\title{
Current Issues in Atopic Comorbidities and Preventing the Atopic March
}

\author{
Eric L. Simpson, MD, MCR, ${ }^{*}$ Lawrence F. Eichenfield, MD, ${ }^{\dagger}$ Charles N. Ellis, MD, ${ }^{\ddagger}$ \\ Anthony J. Mancini, MD, ${ }^{\S}$ and Amy S. Paller, MD"
}

\begin{abstract}
The individual, family, and public health burden of atopic dermatitis (AD) is considerable. The prevalence of $A D$ is high, the signs and symptoms of the disease adversely affect quality of life for patients and their families, and the comorbid conditions associated with $A D$ can increase considerably the negative impact of the disease. These comorbid conditions patients with $A D$ are susceptible to include skin infectious, IgE-mediated diseases, and mental health disorders. New research identifies the skin barrier as not only an important initiator of atopic dermatitis but may even be a site for allergic sensitization to protein antigens. The skin barrier represents a potential new target for novel atopic prevention strategies.
\end{abstract}

Semin Cutan Med Surg 31(suppl 3):S6-S9 (C 2012 Elsevier Inc. All rights reserved.

KEYWORDS: Atopic dermatitis, atopic dermatitis comorbidities, lgE sensitization, transcutaneous

*Associate Professor of Dermatology and Director of Clinical Studies, Oregon Health and Science University, Portland, OR.

$\uparrow$ Professor of Clinical Pediatrics and Medicine (Dermatology) Chief, Pediatric and Adolescent Dermatology, University of California, San Diego School of Medicine, Rady Children's Hospital, San Diego, CA.

₹William B. Taylor, Professor of Clinical Dermatology, Associate Chair, Department of Dermatology, University of Michigan Medical Center, Ann Arbor, MI.

§Professor of Pediatrics and Dermatology Northwestern University Feinberg School of Medicine, and Head, Division of Pediatric Dermatology Children's Memorial Hospital, Chicago, IL.

\|Walter J. Hamlin Professor and Chair, Department of Dermatology, Professor of Pediatrics, Northwestern University Feinberg School of Medicine, Attending Physician, Ann \& Robert Lurie Children's Hospital of Chicago, Chicago, IL

Publication of this CME article was jointly sponsored by the University of Louisville Continuing Health Sciences Education and Global Academy for Medical Education, LLC in affiliation with Skin Disease Education Foundation and is supported by an educational grant from Valeant Pharmaceuticals North America Inc.

Lawrence F. Eichenfield, MD, has served as a consultant for Anacor, Bayer, Onset Therapeutics and as speaker and consultant for Valeant. He has also been an investigator and consultant for Galderma, Leo Pharma as well as an investigator for Amgen, Astellas Pharma US, and Stiefel, A GSK Company.

Charles N. Ellis, MD, has served as a consultant for Galderma, Ferndale Laboratories, Medicis, and Novartis.

Anthony J. Mancini, MD, has served as a consultant for Quinnova and Valeant as well as a speaker and consultant for Galderma.

Amy S. Paller, MD, has received grant research support from Astellas.

Eric L. Simpson, MD, MCR, has served as a consultant, investigator and speaker for Galderma Labs.
A topic dermatitis (AD) is a common disorder, becoming Imore prevalent in developing countries around the world. AD often negatively affects the lives of children and has a major impact on an entire family. AD also predisposes a child to multiple comorbid conditions, such as skin infections, food allergy, asthma, and psychological issues. Because of the high disease prevalence and its impact on patients and families, finding a way to prevent the development of AD has become a focus of intensive research. Additionally, preventing $\mathrm{AD}$ may prevent or reduce the burden of multiple comorbidities that often occur in children who develop AD. Herein, we discuss recent advances regarding AD comorbidities and present a novel skin barrier approach to prevent the atopic march.

The faculty have received an honorarium from Global Academy for Medical Education for their participation in this activity. They acknowledge the editorial assistance of Joanne Still, medical writer, and Global Academy for Medical Education in the development of this continuing medical education journal article. Joanne Still has no relevant financial relationships with any commercial interests.

Address reprint requests to: Eric L. Simpson, MD, MCR, Oregon Health \& Science University Department of Dermatology (CH16D), 3303 S.W. Bond Avenue, Portland, Oregon 97239-4501, Telephone: 503494 2121, Fax: 503494 6907. E-mail: simpsone@ohsu.edu 


\section{Atopic Comorbidities}

\section{Mechanisms of Sensitization}

In the past, the clustering of IgE-mediated conditions led to the assumption that $\mathrm{AD}$ is a disease driven by allergic mechanisms. Indeed, several single nucleotide polymorphisms in genes encoding for immune elements have been found in $\mathrm{AD}$ populations. ${ }^{1}$ However, in light of the robust data implicating the skin barrier gene filaggrin as a strong predictor of $\mathrm{AD}$ development, IgE sensitization and allergy are no longer viewed as causes of AD, but as possible consequences. Protein exposure through a defective skin barrier may be an important mechanism of IgE sensitization in young children, although more direct human data are needed to better understand the importance of transcutaneous sensitization.

Gideon Lack and his team of pediatric allergists conducted one of the first clinical studies to suggest IgE sensitization and allergy may be associated with protein exposure to the skin. This group demonstrated that the strongest predictor of peanut allergy in a cohort of children in the UK was the use in infancy of topical moisturizers containing peanut oil. ${ }^{2}$ This suggested IgE sensitization to peanut proteins was occurring via a transcutaneous route. Lack and colleagues ${ }^{3}$ also demonstrated that food protein concentrations in house dust was strongly associated with an increased risk for peanut allergy.

Studies in both the murine model and in humans support this concept of transcutaneous sensitization. In mouse models, Beck and Leung ${ }^{4}$ showed that applications of protein allergen to abraded skin would produce high levels of IgE to that protein, to a degree greater than that found with sensitization via other routes. In clinical studies, it has been demonstrated that a filaggrin skin barrier gene defect not only increases the risk for $\mathrm{AD}$, but also increases the risk for asthma and allergic sensitization. Most recently, Brown and colleagues ${ }^{5}$ reported that filaggrin defects increase the risk for developing peanut allergy even in the absence of $\mathrm{AD}$ development, further highlighting the importance of the skin barrier in peanut sensitization.

\section{Food Allergy}

The prevalence of food allergy is increased in patients with $\mathrm{AD}$ compared to control populations, although the strength of that risk is not clear. Recent population-based studies show that previous estimates of $30 \%$ to $60 \%$ may be too high, and the risk is probably closer to $15 \% \cdot{ }^{6,7}$ In addition, several papers have been published recently indicating that positive allergy tests in $\mathrm{AD}$ have poor predictive value-that is, the presence of a positive test is not a reliable predictor of either an immediate or delayed clinical reaction..$^{8,9}$ Indeed, an estimated $50 \%$ of the U.S. population will have positive results on an allergy test with no history of allergy. ${ }^{10}$

Recently, the National Institute of Allergy and Infectious Diseases published new guidelines on the diagnosis of food allergy with recommendations for the management of food allergy for patients with AD. ${ }^{11,12}$ Among the changes incorporated by the independent panel of allergists and dermatologists responsible for updating the guidelines is that the di- agnosis of allergy should not be based solely on positive results of allergy testing, but also requires demonstration of an "adverse health effect." The updated NIAID guidelines further state that patients with AD should not be routinely tested for food allergy unless signs are evident of a type 1 reaction (for example, vomiting, urticaria, or angioedema), or unless a patient has been adequately treated with appropriate topical skin care without significant improvement.

\section{Asthma}

There is at least a two-fold increase in asthma risk in children with AD. In addition, asthma severity is known to be worse in patients who also have AD. ${ }^{13}$ The type of asthma that is most common in patients with $\mathrm{AD}$ is allergic asthma. The mechanisms of asthma development in AD are not clear. One possibility is that IgE sensitization, either through immune dysregulation or transcutaneous sensitization, drives allergic asthma. Several studies have suggested that that early exposure to respiratory syncytial virus may dramatically increase the risk for asthma, especially in patients predisposed to atopic disorders. ${ }^{14,15}$

\section{Behavioral/Emotional/ Psychological Issues}

General clinical experience and numerous published studies have shown that children with $\mathrm{AD}$ have an increased prevalence of emotional, behavioral, and psychological issues compared to children without AD. These issues include irritability, fussiness, clingy behavior, restlessness, and scratching the skin as an attention-getting behavior. ${ }^{16}$

In addition, accumulating evidence suggests that children with $\mathrm{AD}$ may be at increased risk for defined mental health disorders, including attention deficit-hyperactivity disorder (ADHD) and autism. Prompted by epidemiologic evidence demonstrating an increased prevalence of asthma in children with ADHD, Schmitt and colleagues ${ }^{17}$ hypothesized that the immunologically related condition of AD might be linked to ADHD symptoms. They studied the possible relationship in a study of more than 1400 German children. These investigators found that the prevalence of ADHD in the subjects with AD was $5.2 \%$, and in controls, it was $3.4 \%$, a statistically significant association (odds ratio, 1.54; $95 \%$ CI, 1.06-2.22; $p=0.02$ ), and possibly indicating a severity-related independent association between AD and ADHD.

However, the authors also point out that their results "require cautious interpretation," noting that even if the association is a real one, it may relate to atopy in general and may not be specific for $\mathrm{AD}$. In addition, they delineate methodologic issues that prevent the establishment of a causative relationship between AD and ADHD. Finally, they indicate that AD-related pruritus, sleep disturbance, and other factors may have been factors in their results.

Our group recently conducted a study of mental health disorders in children with $\mathrm{AD}$ in large populations in the United States that confirm the initial study from Europe. This study demonstrated that pediatric AD is associated with 
ADHD, depression, anxiety, and autism with the greatest risk being associated with more severe skin disease. ${ }^{18}$

A prevailing opinion regarding the underlying mechanisms of behavioral/psychological problems in children with AD is sleep disturbance. Studies have shown that children with AD commonly experience disturbed sleep, both in duration and quality. Disturbed sleep for just a few consecutive nights can manifest in behavior that resembles that associated with ADHD, and children with AD often experience many months and years of poor-quality sleep. It is not yet clear whether the behavioral/psychological problems in children with $\mathrm{AD}$ are fully explained by sleep disturbances, or are the result of other mechanisms entirely.

One other proposed mechanism that may explain-or contribute to-the link between AD and ADHD or autism is systemic inflammation. The theoretical possibility must be considered that cutaneous inflammation may lead to a systemic inflammatory state leading to altered brain development. Children with ADHD and autism have elevated levels of proinflammatory cytokines. ${ }^{19,20}$ Proinflammatory cytokines have been thought to influence brain development, as cytokine receptors can be found in the developing brain.

\section{Infections}

Patients with atopic dermatitis are at increased risk for Staphylococcus aureus colonization and infections. In addition, patients with $\mathrm{AD}$ also get exaggerated presentations of viral infections, particularly to herpes simplex virus (eczema herpeticum), which is associated with a dramatic spreading of viral lesions over the skin, lymphadenopathy, fever, and malaise. Eczema herpeticum can be severe and even life-threatening. Patients with AD also are at increased risk for eczema vaccinatum, a viral skin infection that results from direct vaccination with the smallpox vaccine or, more likely, from close contact with another individual who has been vaccinated.

Understanding of the role and nature of microbial skin infections has been enhanced recently by the work of Capone and colleagues ${ }^{21}$ and of Kong et al, ${ }^{22}$ groups who published groundbreaking articles on the human skin microbiome in infants ${ }^{21}$ and in patients with $\mathrm{AD}$. The skin microbiome is, essentially, the genetic signature of all microorganisms on the skin. These microbiome studies have provided a richer understanding of the microbial diversity and dynamic nature of skin microbes in patients with $\mathrm{AD}$. Kong et $\mathrm{al}^{22}$ demonstrated that flares of AD actually are correlated with a lack of microbial diversity. Staphylococci appear to proliferate in the setting of reduced microbial diversity, although the exact order of events is unclear. Studies of the microbiome provide opportunities for the development of novel strategies in patients in whom recurrent cutaneous infections may be a problem.

\section{Strategies for Prevention}

A systematic review determined that $91 \%$ of previous eczema prevention strategies were based on allergen avoidance or on attempts to alter allergic responses. Some of these examples include the use of hypoallergenic formulas, dietary allergen avoidance, environmental allergen avoidance, and dietary supplements to alter immune reactions in the gut. Unfortunately, after decades of research and more than 100 published studies, no generally accepted prevention strategy for AD exists. ${ }^{23}$

The use of probiotic supplementation and hypoallergenic formulas have shown some recent promising results, but these strategies have not been consistently effective. ${ }^{24,25} \mathrm{In}$ part, this may be because allergic mechanisms are not an important driving factor in atopic dermatitis as previously discussed. Attempting to repair a defective skin barrier early in life represents a new strategy for preventing AD.

In the first study examining a skin barrier approach to $\mathrm{AD}$ prevention, Simpson and colleagues used full-body emollient to prevent the initial flare of AD. This approach was studied in a cohort of 22 babies at high risk for developing eczema (one first-degree relative with a history of atopic disease). ${ }^{26}$ After over 1 year of treatment, the cumulative incidence of $\mathrm{AD}$ was lower than what would be expected using historical controls. These open-label results were recently confirmed in a small, controlled feasibility trial-the Barrier Enhancement for Eczema Prevention (BEEP) study, a collaborative effort between the U.K. and the U.S. involving 124 neonates. ${ }^{27}$ Although the study was not powered to assess efficacy, the results indicated that the approach appears to be safe, feasible, and was associated with significant efficacy in reducing the incidence of $\mathrm{AD}$. The caveats to these results include the fact that it was only a 6-month study and three different emollients were used. However, the results provided a basis on which to further explore this strategy. ${ }^{28}$

\section{Conclusion}

Atopic comorbidities include IgE-mediated diseases, infections, and behavioral and emotional problems. An obvious goal in prevention of AD is to avoid the skin signs and symptoms, but also to prevent these various allergic, infectious, and mental health comorbidities.

Although currently there are no generally accepted, or objectively proven, prevention strategies for $A D$, results from the BEEP feasibility study suggest that measures to protect and enhance the skin barrier from birth may be a promising avenue for future research.

\section{References}

1. Barnes KC: An update on the genetics of atopic dermatitis: Scratching the surface in 2009. J Allergy Clin Immunol 125:16-29, 2010

2. Lack G, Fox D, Northstone K, et al, for the Avon Longitudinal Study of Parents and Children Study Team: Factors associated with the development of peanut allergy in childhood. N Engl J Med 348:977-985, 2003

3. Fox AT, Sasieni P, du Toit G, et al: Household peanut consumption as a risk factor for the development of peanut allergy. J Allergy Clin Immunol 123:417-423, 2009

4. Beck LA, Leung DYM: Allergen sensitization through the skin induces systemic allergic responses. J Allergy Clin Immunol 106:S258-S263, 2000

5. Brown SJ, Kroboth K, Sandilands A, et al: Intragenic copy number 
variation with filaggrin contributes to the risk of atopic dermatitis with a dose-dependent effect. J Invest Dermatol 132:98-104, 2012

6. Bogoniewicz M, Schneider L, Leung D, et al: The allergic profile of inrants in the SAM study: A large longitudinal study of development of asthma and allergies in patients with atopic dermatitis. J Allergy Clin Immunol 119:\$209 (suppl)

7. Silverberg JI, Simpson EL: Predictors of pediatric eczema severity in the United States. J Invest Dermatol 132:S38-S50, 2012

8. Keck LE, Simpson EL, Berry TM, et al: Is food allergy testing reliable in pediatric atopic dermatitis? A population-based study. Chem Immunol Allergy 96:108-112, 2012

9. Fleischer DM, Bock A, Spears GC, et al: Oral food challenges in children with a diagnosis of food allergy. J Pediatr 158:578-583, 2011

10. Arbes SJ Jr, Gergen PJ, Elliott L, et al: Prevalences of positive skin test responses to 10 common allergens in the US population: Results from the third National health and Nutrition Examination Survey. J Allergy Clin Immunol 116:377-383, 2005

11. Boyce JA, Assa'ad A, Burks AW, et al: Guidelines for the diagnosis and management of food allergy in the United States: Report of the NIAIDsponsored expert panel. J Allergy Clin Immunol 126:S1-S58, 2010

12. Burks AW, Tang M, Sicherer S, et al: ICON: Food allergy. J Allergy Clin Immunol 129:906-920, 2012

13. van der Hulst AE, Klip H, Brand PL: Risk of developing asthma in young children with atopic eczema: A systematic review. J Allergy Clin Immunol 120:565-569, 2007

14. Simoes EAF, Carbonell-Estrany X, Rieger CHL, et al: The effect of respiratory syncytial virus on subsequent recurrent wheezing in atopic and nonatopic children. J Allergy Clin Immunol 126:256-262, 2010

15. Sly PD, Kusel M, Holt PG: Do early-life viral infections cause asthma? J Allergy Clin Immunol 125:1202-1205, 2010

16. Chamlin SL: The psychosocial burden of childhood atopic dermatitis. Dermatol Ther 19:104-107, 2006

17. Schmitt J, Romanos M, Schmitt NM, et al: Atopic eczema and attention- deficit/hyperactivity disorder in a population-based sample of children and adolescents. J Am Acad Med 301:724-726, 2009

18. Yaghmaie P, Koudelka CW, Simpson EL: Psychiatric comorbidity in pediatric eczema Apr 2011; 131 (Supplement 1s) 2011 SID Annual Meeting: S1-S173

19. Oades RD, Myint A-M, Dauvermann MR, et al: Attention-deficit hyperactivity disorder (ADHD) and glial integrity: An exploration of associations of cytokines and kynurenine metabolites with symptoms and attention. Behav Brain Functions 6:32, 2010

20. Ashwood P, Wills S, Van de Water J: The immune response in autism: A new frontier for autism research. J Leukoc Biol. 80:1-15, 2006

21. Capone KA, Dowd SE, Stamatas GN, et al: Diversity of the human skin microbiome early in life. J Invest Dermatol 131:2026-2032, 2011

22. Kong H, Segre JA: Skin microbiome: Looking back to move forward. J Invest Dermatol 132:933-939, 2012

23. Simpson EL, Keck LE, Chalmers JR, et al: How should an incident case of atopic dermatitis be defined? A systematic review of primary prevention studies. J Allergy Clin Immunol 130:137-144, 2012

24. van der Aa LB, Heymans HS, van Aalderen WM, et al: Probiotics and prebiotics in atopic dermatitis: Review of the theoretical background and clinical evidence. Pediatr Allergy Immunol 21:e355-e367, 2010

25. Foisy M, Boyle RJ, Chalmers JR, et al: Overview of Reviews: The prevention of eczema in infants and children: An Overview of Cochrane and non-Cochrane reviews. Evid Based Child Health 6:1322-1339, 2011

26. Simpson EL, Berry TM, Brown PA, et al: A pilot study of emollient therapy for the primary prevention of atopic dermatitis. J Am Acad Dermatol 63:587-593, 2010

27. Simpson EL, Chalmers JR, Hanifin JM, et al: Barrier enhancement for eczema prvention-the BEEP feasibility study. Poster presented at: Society for Investigative Dermatology 2012 Annual Meeting, Raleigh, North Carolina; May 9-12, 2012

28. The BEEP study. Available at: www.beepstudy.org. Accessed July 12, 2012 